\title{
Image-Driven Cardiac Left Ventricle Segmentation for the Evaluation of Multiview Fused Real-Time 3-Dimensional Echocardiography Images
}

\author{
Kashif Rajpoot ${ }^{1, *}$, J. Alison Noble ${ }^{1}$, Vicente Grau ${ }^{1}$, \\ Cezary Szmigielski ${ }^{2}$, and Harald Becher ${ }^{2}$ \\ ${ }^{1}$ Institute of Biomedical Engineering, University of Oxford, Oxford, UK \\ ${ }^{2}$ Department of Cardiovascular Medicine, University of Oxford, Oxford, UK \\ \{kashif, noble\}@robots.ox.ac.uk, vicente.grau@oerc.ox.ac.uk, \\ cezary.szmigielski@cardiov.ox.ac.uk, harald.becher@orh.nhs.uk
}

\begin{abstract}
Real-time 3-dimensional echocardiography (RT3DE) permits the acquisition and visualization of the beating heart in 3D. Despite a number of efforts to automate the left ventricle (LV) delineation from RT3DE images, this remains a challenging problem due to the poor nature of the acquired images usually containing missing anatomical information and high speckle noise. Recently, there have been efforts to improve image quality and anatomical definition by acquiring multiple single-view RT3DE images with small probe movements and fusing them together after alignment. In this work, we evaluate the quality of the multiview fused images using an image-driven semiautomatic LV segmentation method. The segmentation method is based on an edge-driven level set framework, where the edges are extracted using a localphase inspired feature detector for low-contrast echocardiography boundaries. This totally image-driven segmentation method is applied for the evaluation of end-diastolic (ED) and end-systolic (ES) single-view and multiview fused images. Experiments were conducted on 17 cases and the results show that multiview fused images have better image segmentation quality, but large failures were observed on ED (88.2\%) and ES (58.8\%) single-view images.
\end{abstract}

\section{Introduction and Literature}

Echocardiography provides a simple, real-time, low-cost, and completely harmless way to assess the cardiac function. It is now possible to capture the $3 \mathrm{D}$ volume sequences of the heart by acquiring real-time 3-dimensional echocardiography (RT3DE) images using a matrix-array ultrasound transducer. Although it has been shown that RT3DE improves reproducibility in comparison to 2D echocardiography [1], it has still not been adopted for routine clinical use for cardiac function analysis. It is expected that reliable and robust automatic methods for left ventricle (LV) endocardial surface extraction will aid the uptake of RT3DE in the clinics.

\footnotetext{
* Kashif Rajpoot is funded by a PhD scholarship from Higher Education Commission, Pakistan.
} 
Cardiac segmentation from RT3DE images is an active area of research and a variety of solutions have been proposed. The major LV segmentation methods for RT3DE images fall into 3 broad categories: edge-driven, region-driven and priormodel driven. In the edge-driven approaches [2,3], a snake-like deformable model [4] is attracted towards the boundary edges usually detected from intensity-gradient based edge detectors. In the region-driven approaches [5,6], image intensities for the LV blood-cavity and myocardium tissue are modeled using a statistical distribution. On the other hand, prior-model driven methods [7,8] construct a statistical model of shape and/or appearance from a training set of RT3DE volumes and use this model to guide LV segmentation. However, it is very difficult to capture the true shape variability of the heart due to its complex nature. Furthermore, it is very challenging to represent the different pathologies in the statistical model. In contrast, both the edge- and region-driven approaches are purely image-driven techniques and they are fully dependent upon the image quality and the anatomical information in the image. This may imply that the segmentation method will be unsuccessful on poor- or average-quality images, due to the problems caused by speckle, missing anatomical boundaries, limited field-of-view (FOV) and intensity dropout (see Fig. 1(b)-(e)). Moreover, quite often a close initialization of the surface is needed in the edge-driven approaches either via registration [2] or manual landmark selection [3].

There have been recent efforts to improve RT3DE image quality by image fusion [9] or compounding [10]. This involves acquiring multiple single-view RT3DE images from different probe positions over the chest cavity and following a 2-step approach to $(i)$ register and $(i i)$ combine them together. Recently, we have developed a wavelet-based multiview RT3DE image fusion method [11] that showed improvements in the signal-to-noise ratio, contrast, and anatomical information. It can also extend the FOV thus permitting a complete 3D coverage of large hearts.

In this work, we use the multiview RT3DE fused images for LV endocardial surface extraction. For this purpose, we introduce an edge-driven level set [4] based LV segmentation framework while the edges are derived from a local-phase inspired feature detector [12] designed for low-contrast echocardiography images. The key contribution of the paper is to objectively assess the quality of multiview fused images against single-view images for automated segmentation.

The paper begins with the details of RT3DE image fusion and the proposed segmentation approach in Section 2. Experimental results and their validation are presented in Section 3. The paper finishes with concluding remarks in Section 4.

\section{Methods}

\subsection{Multiview RT3DE Image Fusion}

We give a brief description of the multiview RT3DE image fusion process here; full details can be found in [11]. The standard single-view RT3DE images are acquired from different transducer positions from the apical view acoustic window (see Fig. 1(a)). The first full-volume image sequence (1) is acquired by placing the transducer probe near the LV apex. Two more full-volume sequences $(2,3)$ are acquired by translating the probe from the apex towards the lateral wall of the $\mathrm{LV}$ by approximately $1 \mathrm{~cm}$ and $2 \mathrm{~cm}$, 
respectively. Another full-volume sequence (4) is acquired by translating the probe from the apex towards the interventricular septum by approximately $1 \mathrm{~cm}$. Finally, two more volume sequences $(5,6)$ are captured by moving the probe one intercostal space above and below the first probe position. In some cases, more than one volume was acquired from the same probe position by a slight angular tilt of the probe.

The acquired multiple single-view images are then aligned using a multiresolution rigid registration algorithm using normalized cross-correlation as a similarity measure and the Powell method as the optimization technique. The first full volume acquired near the apical position is used as the reference volume for registration. Once the image correspondence has been established, the aligned images are then combined together in a way that aims at preserving the salient structures [11]. A wavelet analysis technique is used to decompose each single-view image into its low- and high-frequency components. The fusion is then performed in the wavelet domain, treating the low- and high-frequency wavelet coefficients differently. It was shown in [11] that the fusion improves the anatomical information (measured as the number of relevant features detected in an image) by about 16\%. Fig. 1(b)-(f) show example results of image fusion on 2D image slices.

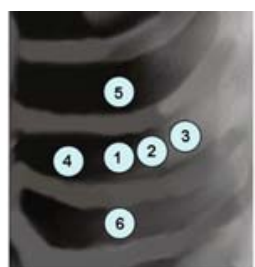

(a)

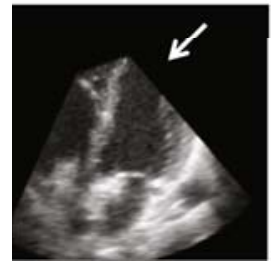

(d)

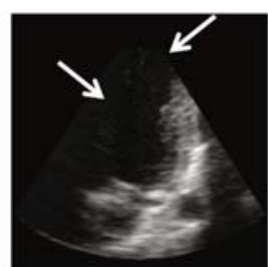

(b)

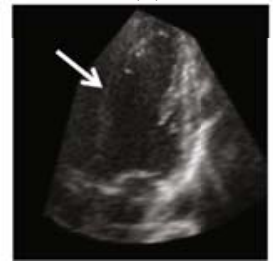

(e)

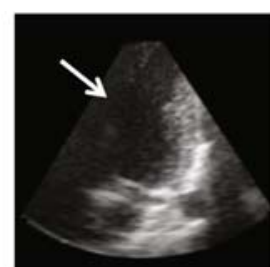

(c)

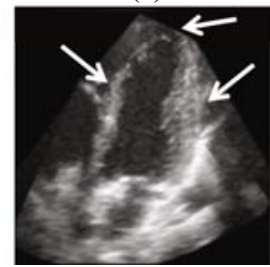

(f)

Fig. 1. Single-view image acquisition and example 2D image results of multiview RT3DE fusion. (a) Image acquisition protocol (probe locations indicated 1 to 6 over chest), (b)-(e) 2D slices from aligned single-view volumes - arrows indicate the missing anatomical information, (f) fused image - arrows depict the filled-in anatomical information due to fusion.

\subsection{Segmentation}

The LV segmentation problem is posed as a deformable surface evolution model [4]:

$$
S_{t}(t, u)=F_{\text {image }}+F_{\text {regularization }}
$$

where $S_{t}(t, u)$ is the surface at time $t$ parameterized by $u, F_{\text {image }}$ is the image based force and $F_{\text {regularization }}$ is the surface regularization force. Following the level set (LS) methods based derivation of Casselles et al. [4], a higher-dimensional implicit 
surface embedding function $\varphi$ can be introduced and surface evolution (1) becomes the solution of a partial differential equation (PDE):

$$
\frac{\partial \varphi}{\partial t}=\alpha g_{I}\|\nabla \varphi\|+\beta \kappa g_{I}\|\nabla \varphi\|+\gamma \nabla g_{I} . \nabla \varphi
$$

where the first term on the right is a balloon force (controlling the growth or shrinkage of the LS), the second term is a curvature-based smoothness term, and the last term is the advection term controlling the attachment of the deformable model to the image edges. In (2), $\alpha, \beta$ and $\gamma$ are the weights for the balloon force, the curvature force $\kappa$ and the advection force, respectively. Here, $g_{I}$ is an edge-indicator function that decreases monotonically with the gradient magnitude of the image $I$ [13]:

$$
g_{I}=f_{\text {map }}\left(\frac{\| \nabla\left(G_{\left.\sigma^{*} I\right) \|}\right.}{\max \| \nabla\left(G_{\left.\sigma^{*} I\right) \|}\right.}\right), \quad f_{\text {map }}(x)=\left[1+(x / v)^{\lambda}\right]^{-1}
$$

where $G_{\sigma} * I$ denotes convolution of image I with a Gaussian kernel of variance $\sigma, v$ is the edge contrast parameter, and $\lambda$ is the edge exponent parameter.

For echocardiography, the intensity-gradient based edge-indicator function $g_{I}$ of (3) is not the best option due to the highly noisy nature of these images. Instead, we adapted the local-phase inspired 3D feature asymmetry $(F A)$ measure [12]. This feature detector is designed for detecting step-like edges (i.e., asymmetric endocardial borders) from low-contrast and noisy echocardiography images. For the computation of the edge-indicator function, we substitute $I$ with $F A$ in (3):

$$
g_{F A}=f_{\text {map }}\left(\frac{\left\|\nabla\left(G_{\sigma} * F A\right)\right\|}{\max \left\|\nabla\left(G_{\sigma} * F A\right)\right\|}\right)
$$

thus our LS surface evolution PDE becomes:

$$
\frac{\partial \varphi}{\partial t}=\alpha g_{F A}\|\nabla \varphi\|+\beta \kappa g_{F A}\|\nabla \varphi\|+\gamma \nabla g_{F A} \cdot \nabla \varphi
$$

To solve (4), we used the Yushkevich et al. implementation of LS methods [13].

\subsection{Post-processing of LV Surface}

The level set based LV endocardial surface extraction method described in the previous section is purely edge-driven. The process works by initializing a sphere inside the LV cavity and allowing it to expand under the influence of balloon force until stopped by the edges. However, there are many false features in the cavity: for example, the edges due to the papillary muscle or the apical region (see Fig. 2(a)). This is a cause of problems in the extraction of the true endocardial surface. We therefore perform a post-processing operation on the LS segmentation. The postprocessing steps are described in the following.

1. Apex point selection. The apex region is a difficult region for an edge-driven endocardial segmentation method because of its very complex shape and lack of boundary definition. Post-segmentation, the middle slice apical 4-chamber plane is presented to the user for manual selection of a point near the apex using a single mouse click. A small sphere is generated at this location having a radius of 3voxels (see Fig. 2(b)), which aids the operation of the next step. 
2. Surface fitting. The LS segmented surface is not smooth because of the papillary muscles and the trabeculae near the endocardium. We perform a hypersurface fitting (tessellation-based linear interpolation) to the LS segmented surface, which now includes the sphere at the apex location from last step (see Fig. 2(c)).

3. Surface smoothness. Commonly, the clinicians expect the endocardial surface to be a smooth surface delineating the endocardium. For this purpose, we perform a Gaussian-smoothing step to smooth the endocardial surface (see Fig. 2(d)).

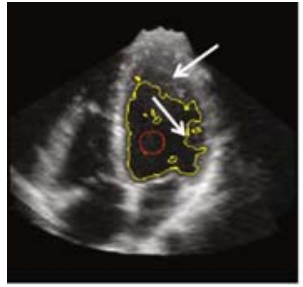

(a)

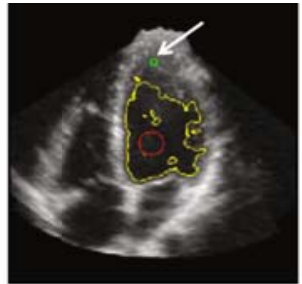

(b)

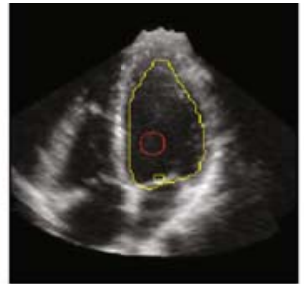

(c)

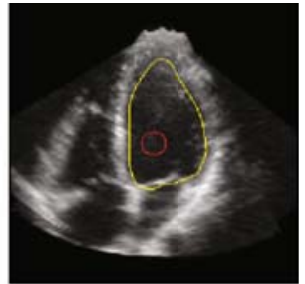

(d)

Fig. 2. Post-processing operations shown on a $2 \mathrm{D}$ end-diastolic slice. Red circle inside the LV cavity is the initial contour placed automatically. (a) LS segmentation (arrows indicate the problematic regions), (b) apex adjustment (arrow indicates the placement of sphere), (c) segmentation after surface fitting, and (d) smoothed final segmentation.

\section{Experimental Results}

\subsection{Data and Experimental Setup}

Volumetric images (17 cases) were obtained from healthy young subjects using the Philips iE33 scanner (Philips Medical Systems, Andover, USA) with a matrix-array transducer (3-5 MHz), acquiring a full-volume sequence by ECG-gating over 4 heartbeats. The usual spatial dimensions are $224 \times 208 \times 208$ voxels. For each case, 3 to 8 single-view images were acquired and fused using the wavelet fusion (see 2.1). To assess the fused image for automatic quantification, the LV segmentation method (see 2.2) was applied to the fused image and one single-view image (the reference volume as in 2.1). For almost all of the subjects, the reference single-view image was of better quality (in terms of anatomical information) than the other single-view images.

End-diastolic (ED) and end-systolic (ES) frames for each dataset were identified by an expert cardiologist. The segmentation method was applied to the ED and ES phases of both fused and reference single-view images. The segmentation was initialized automatically as a sphere of 10 -voxels radius at the centre of the image. The automatic initialization in this way was inside the LV cavity for all the cases except for two ED images, in which case manual initialization was needed. The successful convergence (the ability to reach near the LV endocardial border) of the segmentation method was quantified and the clear failures of the method were visually classified according to the possible causes: ( $i$ ) LV cavity speckle noise, (ii) boundary leakage due to insufficient boundary information at the endocardial border, and (iii) both leakage and noise. To validate the successful segmentation cases, the fused images were also manually segmented by an expert cardiologist using 
commercial software (CardioView, TomTec, Germany) to obtain measurements for ED volume (EDV), ES volume (ESV), and ejection fraction (EF). LV trabeculations and papillary muscles were included within the LV cavity. In addition, magnetic resonance images (MRI) from all the cases were acquired for reference and the EDV, ESV, and EF measurements were calculated by an expert cardiologist.

\subsection{Results}

Fig. 3 shows example segmentation results on both single-view and fused images, demonstrating the lack of sufficient information in the single-view images for a successful segmentation. On the other hand, the same segmentation method works successfully on a fused image. Table 1 summarizes the segmentation failures for single-view images, while there were no failures on the fused images. For single-view images, the algorithm failed in most cases at both end-diastole (88.2\%) and endsystole (58.8\%). There were fewer failures at ES because the myocardium is thicker during this phase, providing better boundary definition further demonstrated by only $30 \%$ failures at ES due to leakage or leakage and noise compared to $60 \%$ failures at ED. The absolute difference in EDV, ESV, and EF measurements is given in Table 2. The RT3DE EDV and ESV are underestimated compared to MRI, which has been reported before [1]. The EDV differences with automatic RT3DE $(55.9 \pm 23.1)$ and manual RT3DE $(64.5 \pm 19.9)$ in comparison to MRI are considerably underestimated, probably due to large MRI EDVs $(205.8 \pm 19.7)$ because the scanned subjects were young athletes having large hearts. However, the clinically important EF measure is within the known reproducibility range [1] (see Table 2). Fig. 4 presents Bland-Altman analysis demonstrating that there is a good statistical agreement between the automatic and manual RT3DE and MRI measurements.

Table 1. Failure of segmentation on single-view images (17 datasets) and its quantification. There were no failures on multiview fused images.

\begin{tabular}{c|c|c} 
& End-diastolic phase & End-systolic phase \\
\hline Total Failure & $\mathbf{8 8 . 2} \%(\mathbf{1 5})$ & $\mathbf{5 8 . 8} \%(\mathbf{1 0})$ \\
\hline Cavity noise & $40 \%(6)$ & $70 \%(7)$ \\
\hline Boundary leakage & $13 \%(2)$ & $20 \%(2)$ \\
\hline Leakage + noise & $47 \%(7)$ & $10 \%(1)$ \\
\hline
\end{tabular}

Table 2. Absolute differences in quantification of EDV, ESV, and EF (17 datasets). AEcho Automatic measurements. MEcho - Manual measurements. MRI - MRI measurements. The differences are given as average \pm standard deviation.

\begin{tabular}{c|c|c|c} 
& AEcho vs. MEcho & MEcho vs. MRI & AEcho vs. MRI \\
\hline EDV (mL) & $14.6 \pm 6.9$ & $64.5 \pm 19.9$ & $55.9 \pm 23.1$ \\
\hline ESV $(\mathrm{mL})$ & $9.1 \pm 6.2$ & $20.0 \pm 9.7$ & $18.0 \pm 11.5$ \\
\hline EF $(\%)$ & $5.9 \pm 4.9$ & $5.0 \pm 7.0$ & $8.3 \pm 4.9$ \\
\hline
\end{tabular}




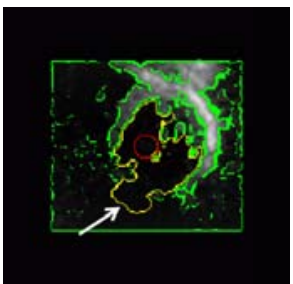

(a) single-view SAX

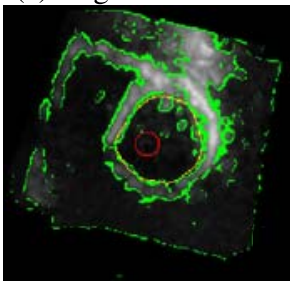

(d) fused SAX
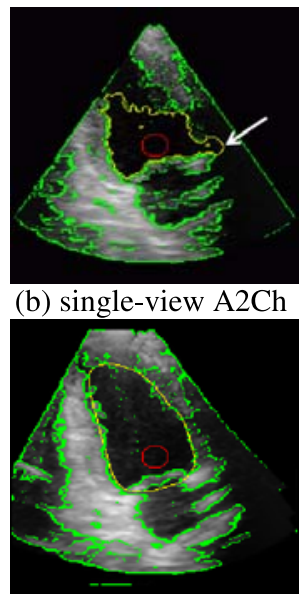

(e) fused $\mathrm{A} 2 \mathrm{Ch}$

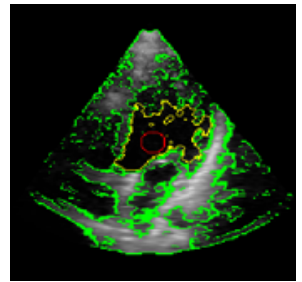

(c) single-view A4Ch

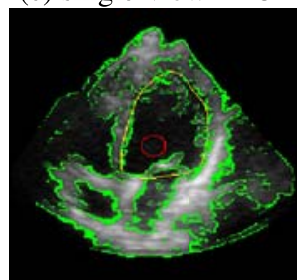

(f) fused $\mathrm{A} 4 \mathrm{Ch}$

Fig. 3. Segmentation results on orthogonal planes. Red - automatic initialization. Green - edgeindicator features. Yellow - segmentation result. SAX - Short-axis plane. A 2Ch - Apical 2chamber plane. A4Ch - Apical 4-chamber plane. Arrows indicate failure of the segmentation due to leakage. No post-processing was performed on the single-view image due to failure.
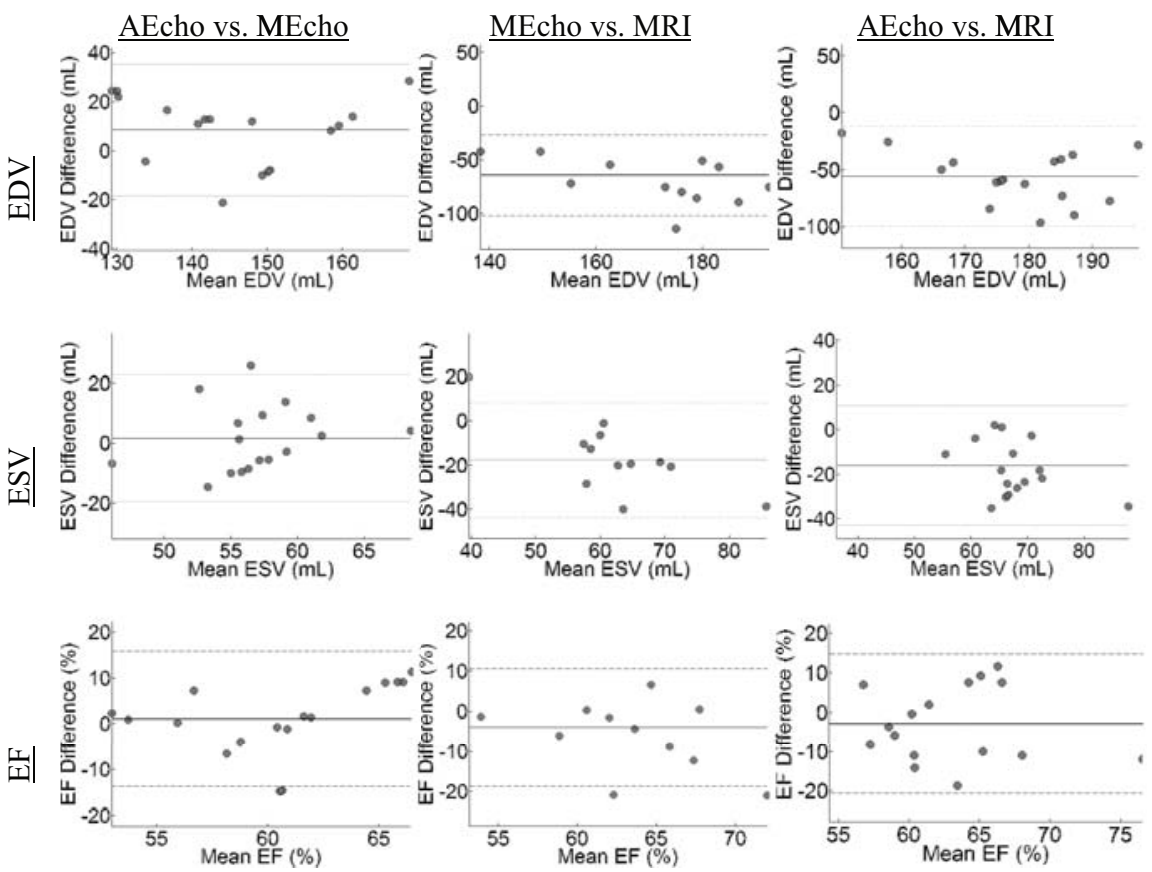

Fig. 4. Bland-Altman analysis of EDV (mL), ESV (mL), and EF (\%). Average difference and $95 \%$ confidence interval are displayed as solid and dashed lines, respectively. 


\section{Conclusions}

This work introduced an edge-driven LS segmentation method while using localphase derived edges as the driving force. The segmentation method was then applied for the evaluation of multiview fused RT3DE images against the conventional singleview RT3DE images. The experiments indicate that a relatively simple segmentation method does much better on the multiview fused than in single-view images. Moreover, the clinical measures derived from automatic segmentation results on fused images are very close to manually computed measures. The results thus demonstrate that the fused images are better suited for automated segmentation because of improved anatomical definition and noise reduction due to fusion.

\section{References}

1. Jenkins, C., Bricknell, K., Hanekom, L., Marwick, T.: Reproducibility and Accuracy of Echocardiographic Measurements of Left Ventricular Parameters Using Real-Time 3-D Echocardiography. Journal of the American College of Cardiology 44 (2004)

2. Zagrodsky, V., Walimbe, V., Castro-Pareja, C., Qin, J.X., Song, J.-M., Shekhar, R.: Registration-Assisted Segmentation of Real-Time 3-D Echocardiographic Data Using Deformable Models. IEEE Transactions on Medical Imaging 24, 1089-1099 (2005)

3. Corsi, C., Saracino, G., Sarti, A., Lamberti, C.: Left Ventricular Volume Estimation for Real-Time Three-Dimensional Echocardiography. IEEE Trans. MI 21, 1202-1208 (2002)

4. Casselles, V., Kimmel, R., Sapiro, G.: Geodesic Active Contours. IJCV 22, 61-79 (1997)

5. Angelini, E.D., Homma, S., Pearson, G., Holmes, J.W., Laine, A.F.: Segmentation of RT3D Ultrasound for Quantification of Ventricular Function: A Clinical Study on Right and Left Ventricles. Ultrasound in Med. \& Biol. 31, 1143-1158 (2005)

6. Zhu, Y., Papademetris, X., Sinusas, A.J., Duncan, J.S.: Segmentation of myocardial volumes from real-time 3D echocardiography using an incompressibility constraint. In: Ayache, N., Ourselin, S., Maeder, A. (eds.) MICCAI 2007, Part I. LNCS, vol. 4791, pp. 44-51. Springer, Heidelberg (2007)

7. Stralen, M.V., Leung, K.Y.E., Voormolen, M.M., et al.: Automatic Segmentation of the Left Ventricle in 3D Echcoardiography Using Active Appearance Models. In: IEEE Ultrasonics Symposium, pp. 1480-1483 (2007)

8. Hansegard, J., Orderud, F., Rabben, S.I.: Real-Time Active Shape Models for Segmentation of 3D Cardiac Ultrasound. Computer Analysis of Images \& Patterns (2007)

9. Soler, P., Gerard, O., Allain, P., Saloux, E., Angelini, E., Bloch, I.: Comparison of Fusion Techniques for 3D+T Echocardiography Acquisitions from Different Acoustic Windows. Computers in Cardiology, 141-144 (2005)

10. Grau, V., Noble, J.A.: Adaptive multiscale ultrasound compounding using phase information. In: Duncan, J.S., Gerig, G. (eds.) MICCAI 2005. LNCS, vol. 3749, pp. 589596. Springer, Heidelberg (2005)

11. Rajpoot, K., Noble, J.A., Grau, V., Szmigielski, C., Becher, H.: Multiview RT3D Echocardiography Image Fusion. In: Functional Imaging \& Modeling of Heart. LNCS, vol. 5528, pp. 134-143. Springer, Heidelberg (2009)

12. Rajpoot, K., Grau, V., Noble, J.A.: Local-phase Based 3D Boundary Detection using Monogenic Signal and its Application to RT3D Echocardiography Images. In: ISBI (2009)

13. Yushkevich, P.A., Piven, J., Hazlett, H.C., Smith, R.G., Ho, S., Gee, J.C., Gerig, G.: Userguided 3D Active Contour Segmentation of Anatomical Structures: Significantly Improved Efficiency and Reliability. NeuroImage 31 (2006) 